\title{
Rank of Quantized Universal Enveloping Algebras and Modular Functions
}

\author{
Shahn Majid ${ }^{1 \star}$ and Ya. S. Soibelman ${ }^{2}$ \\ 1 Department of Applied Mathematics and Theoretical Physics, University of Cambridge, \\ Cambridge CB3 9EW, UK \\ ${ }^{2}$ Computer Centre, University of Rostov, Rostov-on-Don, USSR
}

Received April 3, 1990; in revised form August 28, 1990

\begin{abstract}
We compute an intrinsic rank invariant for quasitriangular Hopf algebras in the case of general quantum groups $U_{q}(g)$. As a function of $q$ the rank has remarkable number theoretic properties connected with modular covariance and Galois theory. A number of examples are treated in detail, including $\operatorname{rank}\left(U_{q}(s u(3))\right)$ and rank $\left(U_{q}\left(e_{8}\right)\right)$. We briefly indicate a physical interpretation as relating ChernSimons theory with the theory of a quantum particle confined to an alcove of $g$.
\end{abstract}

\section{Introduction}

Quasitriangular Hopf algebras (or "quantum groups") are a close generalization of groups. In particular, for finite-dimensional quasitriangular Hopf algebras $H$ there is a natural invariant, $\operatorname{rank}(H)$, arising out of category theory as a natural generalization of the order, $|G|$, of a finite group $G[19,18]$. The definition is recalled in the preliminaries below. Moreover, the rank extends in a natural way to the infinite-dimensional quantum groups $U_{q}(g)$. It was computed explicitly for $g=s u(2)$ as [19],

$$
\operatorname{rank}\left(U_{q}(s u(2))\right)=\frac{1+2 \sum_{n \in \mathbb{N}} q^{-(1 / 2) n^{2}}}{1-q^{-2}} .
$$

The numerator here is a theta function $\theta\left(q^{-1 / 2}\right)$ and has remarkable modular transformation properties under $\tau \rightarrow-\frac{1}{\tau}$. Here $q=e^{2 \pi u \tau}$ and explicitly, $\theta\left(e^{-\pi / \tau}\right)=$ $(i \tau)^{1 / 2} \theta\left(e^{\pi \tau \tau}\right)$. Because rank is an invariant, this suggests the possibility of simple transformation properties of the algebra $U_{q}(s u(2))$ as $q$ undergoes a modular

\footnotetext{
* SERC Research Fellow and Drapers Fellow of Pembroke College, Cambridge
} 
transformation. Our goal in this paper is to show that these results are not peculiar to $U_{q}(s u(2))$ but hold to some extent for more general quantum groups.

We remark that quantum groups are known to be related to the chiral algebras of certain conformal field theories. For example, one well known point of similarity is between quantum groups at roots of unity and affine Kac-Moody Lie algebras. For a further point of interest see [16]. Since modular transformations play an important role in such conformal field theories, we might expect that they also have a role for quantum groups [1]. The modular transformation properties of $\operatorname{rank}\left(U_{q}(s u(2))\right)$ and their analogues below are nevertheless surprising. This is because it is the parameter $q$ itself that is transformed: $q$ corresponds in the usual point of view to the level of the Kac-Moody algebra and is not something to which we would expect to apply modular transformations in physics. See however, the discussion in the concluding section.

The results of the present paper are as follows: in Sect. 2 we formulate some expectations regarding $\operatorname{rank}\left(U_{q}(g)\right)$ as a function of $q$. These motivate the main results of the paper. We show in Sect. 3 that for general complex simple $g$, rank $\left(U_{q}(g)\right)$ takes the form of a $\theta$ function associated to the weight lattice of $g$. We also show that $\operatorname{rank}\left(U_{q}(g)\right)$ has an infinite product representation that vanishes on $q$ any rational root of unity. Finally, in Sect. 4 we look at a number of examples in detail. Among these, we find that the functional dependence of $\operatorname{rank}\left(U_{q}(s u(3))\right)$ on $q$ is not evidently that of a modular function but nevertheless has interesting number-theoretic multiplicativity properties of similar character. We conclude with some remarks about a possible physical interpretation.

Preliminaries. $\mathbb{N}$ denotes natural numbers excluding 0 . We denote by $\mathbb{Z}_{+}$the natural numbers including 0 . We adopt the usual notations for Hopf algebras and quantum groups over $\mathbb{C}$ or over formal power series in an indeterminate. Thus a Hopf algebra $H$ is an algebra equipped with a coproduct $\Delta$, counit $\varepsilon$ and antipode $S$. It is quasitriangular (a "quantum group" in the strict sense) if there is an element $\mathscr{R} \in H \otimes H$ obeying certain axioms $[5]$, namely, $(\Delta \otimes \mathrm{id})(\mathscr{R})=\mathscr{R}_{13} \mathscr{R}_{23},(\mathrm{id} \otimes \Delta)(\mathscr{R})=$ $\mathscr{R}_{13} \mathscr{R}_{12}$ and $\tau \circ \Delta h=\mathscr{R}(\Delta h) \mathscr{R}^{-1}$ for all $h \in H$. Here $\mathscr{R}$ is assumed invertible, $\tau$ is the twist map and $\mathscr{R}_{12}=\mathscr{R} \otimes 1$ etc. See $[19,5]$ for an introduction.

The representations of $H$ as an algebra form a quasitensor category (also known as "quasisymmetric" or "braided monoidal" $[13,9])$. See for example $[19$, Sect. 7$]$. This means that there is a tensor product, commutative and associative up to isomorphism, in a compatible way. The tensor product of modules $\otimes$ is given via the coproduct of $H$, the associativity by vector space associativity and the quasisymmetry $\Psi_{V_{1}, V_{2}}: V_{1} \otimes V_{2} \rightarrow V_{2} \otimes V_{1}$ by $\Psi_{V_{1}, V_{2}}\left(v_{1} \otimes v_{2}\right)=$ $\Sigma \mathscr{R}^{(2)} \cdot v_{2} \otimes \mathscr{R}^{(1)} \cdot v_{1}$. Here $v_{1} \otimes v_{2} \in V_{1} \otimes V_{2}$ and $\mathscr{R}=\Sigma \mathscr{R}^{(1)} \otimes \mathscr{R}^{(2)}$.

In quasitensor categories, as for tensor categories [4], we can ask for the existence of an "internal hom" object for each pair of objects $V_{1}, V_{2}$ such that $\operatorname{Mor}\left(V_{3} \otimes V_{1}, V_{2}\right) \stackrel{\ominus}{\cong} \operatorname{Mor}\left(V_{3}, \underline{\operatorname{Hom}}\left(V_{1}, V_{2}\right)\right)$ for all objects $V_{3}$ by functorial isomorphisms $\theta$ (Mor denotes morphisms in the category). In this case one can show that there are morphisms

$$
\underline{\operatorname{Hom}}\left(V_{1}, W_{1}\right) \otimes \underline{\operatorname{Hom}}\left(V_{2}, W_{2}\right) \stackrel{\psi_{1}}{\longrightarrow} \underline{\operatorname{Hom}}\left(V_{1} \otimes V_{2}, W_{1} \otimes W_{2}\right), \quad V \stackrel{\psi_{2}}{\longrightarrow} V^{* *},
$$

where $V^{*}=\underline{\operatorname{Hom}}(V, \underline{1})$ and $\underline{1}$ is the unit object (in our case, the trivial representation). If these maps are isomorphisms then the category is said to be rigid cf. 
[4]. The finite-dimensional modules of $H$ are rigid with $\operatorname{Hom}\left(V_{1}, V_{2}\right)=$ $\operatorname{Lin}\left(V_{1}, V_{2}\right)$ an $H$-module via $(h \cdot f)\left(v_{1}\right)=\Sigma h_{(1)} \cdot\left(f\left(S h_{(2)} \cdot v_{1}\right)\right)$ for all $h \in H$, $f \in \operatorname{Hom}\left(V_{1}, V_{2}\right)$ and $v_{1} \in V_{1}$. The notation here is $\Delta h=\Sigma h_{(1)} \otimes h_{(2)}$. The maps $\psi_{1}, \psi_{2}$ and their inverses were given explicitly in [19, Sect. 7.3.7].

In a rigid tensor or quasitensor category there is a map, Trace:Mor $(V, V) \rightarrow$ Mor $(\underline{1}, \underline{1})$ coming from $\operatorname{Mor}(\underline{1}$,$) applied to$

$$
\underline{\operatorname{Hom}}(V, V) \stackrel{\psi_{1}^{-1}}{\longrightarrow} \underline{\operatorname{Hom}}(V, \underline{1}) \otimes \underline{\operatorname{Hom}}(\underline{1}, V) \stackrel{e v}{\longrightarrow} \underline{1} .
$$

Here we identify $\underline{\operatorname{Hom}}(\underline{1}, V)=V$ and $e v=\theta^{-1}\left(\operatorname{id}_{V^{*}}\right)$. Every object in the category therefore has a natural intrinsic rank or "dimension" defined as Trace of id $V$ [4]. $\operatorname{rank}(V)$ has some natural properties and in the tensor case is multiplicative [4]. We have followed here the treatment of rank via Hom analogous to that of [4].

In our case, in the category of $H$-modules and over $\mathbb{C}$, we identify $\operatorname{Mor}(\underline{1}, \underline{1})=\mathbb{C}$ and normalize so that $\operatorname{rank}(\underline{1})=1$. Then [19],

Proposition 1.1 [19]. Let $H$ be a quasitriangular Hopf algebra and $V$ an H-module. The intrinsic category theoretic rank of $V$ is

$$
\operatorname{rank}(V)=\operatorname{Tr}_{V}(\underline{u}), \quad \underline{u}=\sum\left(S \mathscr{R}^{(2)}\right) \mathscr{R}^{(1)} .
$$

In particular, $H$ acts on itself in the left regular representation. Hence,

Definition 1.2 [19]. Let $H$ be a finite-dimensional quasitriangular Hopf algebra. We define $\operatorname{rank}(H)$ as the intrinsic rank of $H$ as an $H$-module by left multiplication.

Some general results about $\operatorname{rank}(H)$ are in [18] along with a treatment of the case when $H$ is a quantum double. For the infinite-dimensional case of interest in the present paper we adopt,

Definition $1.3 \mathrm{cf}$ [ [19]. Let $P_{+}$denote the set of dominant weights of $g$ and $V(\Lambda)$ the irreducible highest weight $U_{q}(g)$-module with highest weight $\Lambda \in P_{+}$. We define

$$
\operatorname{rank}\left(U_{q}(g)\right)=\sum_{\Lambda \in \mathcal{P}_{+}}(\operatorname{dim} V(\Lambda)) \operatorname{rank}(V(\Lambda))
$$

as a formal power-series in $q$.

This is motivated by the Peter-Weyl theorem for the decomposition of the left regular representation of a compact Lie group with Lie algebra $g$, as follows. In general the $g$-modules $V(\Lambda)$ are also $U_{q}(g)$-modules and in general have the same multiplicities as their classical counterparts. In particular (with suitable completions and for a restricted range of $q$ ) the left regular representation decomposes in the same way as $\bigoplus_{\Lambda \in P_{+}}(\operatorname{dim} V(\Lambda)) V(\Lambda)$. Hence Definition 1.3 follows formally from Definition 1.2 applied in an infinite-dimensional setting. This is the strategy adopted for defining $\operatorname{rank}\left(U_{q}(s u(2))\right)$ in [19].

The conventions for the quantum groups $U_{q}(g)$ will be as follows. Let $t$ be a Cartan sub-algebra of $g$ and $\alpha_{i} \in t^{*}$ a system of positive simple roots. Here $i$ runs from 1 up to $l=\operatorname{rank}(g)$. It is possible to associate to this root system generators $\left\{q^{ \pm H_{i} / 2}, X_{i}, X_{-i}\right\}$ generating a Hopf algebra over $\mathbb{C}[12]$. We had this in mind with a suitable topological completion in the derivation of Definition 1.3. However, for our present purposes we can also follow [5] and define $U_{q}(g)$ over formal power-series $\mathbb{C}[[\hbar]]$ (with $q=e^{\hbar / 2}$ ). Then the generators are $\left\{H_{i}, X_{i}, X_{-i}\right\}$. As 
elements of a vector space we identify them with the corresponding elements of a Cartan-Weyl basis of the $g$. In particular we have $K\left(H_{i}, h\right)=\alpha_{i}(h)$ for all $h \in t$, and where $K$ denotes the Killing form. The inverse of the Killing form defines a symmetric bilinear form (,) on $t^{*}$ in the usual way and we let $a_{i j}=\left(\check{\alpha}_{i}, \alpha_{j}\right)$, where $\check{\alpha}_{i}=2 \alpha_{i} /\left(\alpha_{i}, \alpha_{i}\right)$. We assume that the Cartan matrix has $a_{i j}<0$ for $i \neq j$. We do not, however, make use of the ordinary Lie bracket structure on the vector space of $g$ but rather define the relations of $U_{q}(g)$ as

$$
\begin{aligned}
& {\left[H_{i}, X_{ \pm j}\right]= \pm\left(\alpha_{i}, \alpha_{j}\right) X_{ \pm j}, \quad\left[X_{i}, X_{-j}\right]=\delta_{i j} \frac{q^{H_{i}}-q^{-H_{i}}}{q-q^{-1}}} \\
& \sum_{k=0}^{1-a_{i j}}(-1)^{k}\left[\begin{array}{c}
1-a_{i j} \\
k
\end{array}\right]_{q \|^{\left\|\alpha_{i}\right\|^{2} / 2}} X_{ \pm i}^{1-a_{i j}-k} X_{ \pm j} X_{ \pm i}^{k}=0, \quad \forall i \neq j .
\end{aligned}
$$

Here $[n]_{q}=\frac{q^{n}-q^{-n}}{q-q^{-1}}$ is used to define the $q$-binomial coefficient. The coproduct is
defined by

$$
\Delta H_{i}=H_{i} \otimes 1+1 \otimes H_{i}, \quad \Delta X_{ \pm i}=X_{ \pm i} \otimes q^{H_{i} / 2}+q^{-H_{i} / 2} \otimes X_{ \pm i},
$$

the counit by $\varepsilon\left(H_{i}\right)=\varepsilon\left(X_{ \pm i}\right)=0$ and the antipode acting on the generators by -1 times conjugation by $q^{\check{\rho}}$, where

$$
\check{\rho}=\frac{1}{2} \sum_{\alpha>0} H_{\alpha},
$$

where $H_{\alpha}$ is the element of the vector space $t$ defined by $K\left(H_{\alpha}, h\right)=\alpha(h)$ for all $h \in t$. The universal quasitriangular element $\mathscr{R}$ for $U_{q}(g)$ is computed explicitly in [22]. We let $\rho=\frac{1}{2} \sum_{\alpha>0} \alpha$ so that $\rho=K(\check{\rho}, \quad)$.

\section{Rank Formulate}

This section motivates the following conjecture for $\operatorname{rank}\left(U_{q}(g)\right)$. This in turn motivates some of the results that follow in later sections. We sketch an heuristic or formal proof based on Hopf-algebraic considerations.

Conjecture 2.1. $\operatorname{rank}\left(U_{q}(g)\right)$ vanishes for all $q$ a primitive root of unity with the possible exception of $q$ for which $q^{\left\|\alpha_{i}\right\|^{2}}=1$ for all simple roots $\alpha_{i}$. For general $q$,

$$
\operatorname{rank}\left(U_{q}(g)\right)=\frac{\prod_{n=1}^{\infty}\left(1-q^{-n}\right) P_{n}(q)}{\prod_{\alpha>0}\left(1-q^{-2(\alpha, \rho)}\right)},
$$

where $P_{n}(q)$ are polynomials in a rational power of $q$.

Proof. The arguments for this are as follows. As we approach a primitive root of unity, representations of $U_{q}(g)$ become unfaithful since it can be shown that the action of $X_{ \pm i}$ to a certain power $k$, say, is zero. Since this happens in a uniform way for all representations we can expect that $\operatorname{rank}\left(U_{q}(g)\right)$ evaluated at a root of unity coincides with $\operatorname{rank}\left(U_{q}(g)^{\prime}\right)$. Here we suppose that $U_{q}(g)^{\prime}$ is a finite- 
dimensional quasitriangular Hopf algebra over $\mathbb{C}$ obtained from $U_{q}(g)$ by including relations of the form $X_{ \pm i}^{k}=0$. This is well understood only for $g=s u(2)$, but something along these lines seems plausible for general $g$. Except for certain values of $q$ this finite-dimensional Hopf algebra $U_{q}(g)^{\prime}$ has antipode with square not equal to one. $S^{2}$ is given by conjugation by $q^{2 \check{\rho}}$ so the exceptional values are such that $q^{2\left(\alpha_{i}, \rho\right)}=1$ for all $i$. Note that $2\left(\alpha_{i}, \rho\right)=\left\|\alpha_{i}\right\|^{2}$ as $\left(\check{\alpha}_{i}, \rho\right)=1$. Hence by recent work of Larson and Radford [15], $U_{q}(g)^{\prime}$ is not semisimple at the non-exceptional values of $q$. However, it was shown in [18] that (in our case over $\mathbb{C}$ ), the rank of a non-semisimple finite-dimensional Hopf algebra is zero: $\operatorname{rank}\left(U_{q}(g)^{\prime}\right)=0$. If the first part of the conjecture is true then the second part may also be expected for sufficiently well behaved formal power-series $\operatorname{rank}\left(U_{q}(g)\right)$. The denominator here is a simple expression in terms of the root system that vanishes whenever $q^{\left\|\alpha_{i}\right\|^{2}}=1$ for all simple roots $\alpha_{i}$.

This was already found for $g=s u(2)$ in the form of a theta function;

$$
\operatorname{rank}\left(U_{q}(s u(2))\right)=\frac{\prod_{n=1}^{\infty}\left(1-q^{-n}\right)\left(1+q^{-n+1 / 2}\right)^{2}}{1-q^{-2}}
$$

via the Jacobi triple product identity for theta functions. This vanishes at all roots of unity (including at the exceptional value of $q$, namely $q^{2}=1$ ). The conjecture will be verified for $g=s u(3), s u(4)$ in Sect. 4.

To begin the computation of $\operatorname{rank}\left(U_{q}(g)\right)$ we make use of a result of Drinfeld:

Proposition 2.2 [6]. Let $V$ be an irreducible $U_{q}(g)$-module generated by an element $v$ such that $X_{i} v=0$ for all $X_{i}$ and $h v=\Lambda(h) v$ for all $h \in t$ and some $\Lambda \in t^{*}$. Then acting on $V$,

$$
\underline{u}=q^{2 \check{\rho}} q^{-(\Lambda, \Lambda+2 \rho)}
$$

where $\rho=\frac{1}{2} \sum_{\alpha>0} \alpha$.

Proof [6]. The element $\underline{u}$ as in Proposition 1.1 can be shown to implement the square of the antipode by conjugation (this is a general feature of quasitriangular Hopf algebras). Hence $q^{-2 \tilde{\rho}} \underline{u}$ is central. Hence in an irreducible representation it is proportional to the identity, hence can be computed on any vector in $V$. Computation on the vector $v$ is particularly easy because the $X_{i}$ vanish so that only the lowest part of the series for $\mathscr{R}$ contributes. This lowest part involves only the $H_{i}$ as in [5]. Again, on $v$ the Cartan subalgebra $t$ acts by $\Lambda$, immediately giving the result.

Let $V$ be a highest weight $U_{q}(g)$-module. The weight spaces $V_{\lambda}$ are defined in the usual way as

$$
V_{\lambda}=\{v \in V \mid h v=\lambda(h) v, \forall h \in t\} .
$$

The set $P(V)=\left\{\lambda \in t^{*} \mid V_{\lambda} \neq 0\right\}$ is the set of weights of $V$ and $V$ is a direct sum of the $V_{\lambda}$ with multiplicity $\operatorname{dim} V_{\lambda}$. Denoting the set of weights of $V$ as $P(V)$, the character of $V$ is defined as $\operatorname{ch}(V)=\sum_{\lambda \in P(V)}\left(\operatorname{dim} V_{\lambda}\right) q^{\lambda}$, where $q^{\lambda}$ (or more usually $\left.e^{\lambda}\right)$ is a formal exponential. Hence 
Corollary 2.3. Let $P_{+}$be the set of dominant weights, i.e. $P_{+}=\left\{\Lambda \in t^{*} \mid\left(\Lambda, \check{\alpha}_{i}\right) \in\right.$ $\left.\mathbb{Z}_{+}, \forall i=1, \ldots, \operatorname{rank}(g)\right\}$. Then

$$
\operatorname{rank}\left(U_{q}(g)\right)=\sum_{\Lambda \in P_{+}}(\operatorname{dim} V(\Lambda)) q^{-(\Lambda, \Lambda+2 \rho)} \operatorname{ch}(V(\Lambda))(2 \rho) .
$$

This version underlies a geometric picture of rank. Also, we can immediately write this in terms of $W$, the Weyl group of $g$. $W$ acts on $t^{*}$ as a group of isometries.

Proposition 2.4. Let $W$ be the Weyl group of $g$. Then

$$
\operatorname{rank}\left(U_{q}(g)\right)=\sum_{\Lambda \in P_{+}}(\operatorname{dim} V(\Lambda)) \frac{q^{-\|\Lambda+\rho\|^{2}}}{q^{-\|\rho\|^{2}}}\left(\frac{J(\Lambda+\rho)}{J(\rho)}\right)(2 \rho),
$$

where $J(\mu)(v)=\left(\sum_{w \in W}(\operatorname{det} w) q^{w \mu}\right)(v)=\sum_{w \in W}(\operatorname{det} w) q^{(w \mu, v)}$.

Proof. Use the Weyl character formula in Corollary 2.3.

This version makes clear a general comparison with the Macdonald identities [17],

$$
\sum_{\lambda \in M} Q^{\left(\|\lambda+\rho\|^{2}\right) / 2 g_{c}} \frac{J(\lambda+\rho)}{J(\rho)}=Q^{d / 24} \prod_{n=1}^{n=\infty} P\left(Q^{n}\right),
$$

where $P(Q)=(1-Q)^{l} \prod_{\alpha}\left(1-Q q^{\alpha}\right), M$ is a certain sublattice of the weight lattice and $g_{c}$ is a constant. In the present context, $\|\rho\|^{2} / 2 g_{c}=d / 24, d=\operatorname{dim} g$ and $l=\operatorname{rank} g$. Evaluating on $2 \rho$ gives an expression on the left similar to that for $\operatorname{rank}\left(U_{q}(g)\right)$ in Proposition 2.4 , but without the $\operatorname{dim} V(\Lambda)$ present there. On the other hand, evaluating the identity on 1 gives [17]

$$
\sum_{\lambda \in M}(\operatorname{dim} V(\lambda)) Q^{\left(\|\lambda+\rho\|^{2}\right) / 2 g_{c}}=\eta(Q)^{d},
$$

where $\operatorname{dim}(V(\lambda))=\prod_{\alpha>0} \frac{(\lambda+\rho, \alpha)}{(\rho, \alpha)}$ and $\eta(Q)=Q^{1 / 24} \prod_{n=1}^{\infty}\left(1-Q^{n}\right)$ is the Dedekind $\eta$-function. This comparison with the Macdonald identities suggests that $\operatorname{rank}\left(U_{q}(g)\right)$ too should have a similar infinite product representation, as expected from Conjecture 2.1 .

We conclude with a more explicit version of Proposition 2.4, suitable for direct computations.

Proposition 2.5. Let $[n]_{q}=\frac{q^{n}-q^{-n}}{q-q^{-1}}$. Then

$$
\operatorname{rank}\left(U_{q}(g)\right)=\sum_{\Lambda \in \boldsymbol{P}_{+}} \frac{q^{-\|\Lambda+\rho\| 2}}{q^{-\|\rho\|^{2}}}\left(\prod_{\alpha>0} \frac{(\alpha, \Lambda+\rho)}{(\alpha, \rho)}\right)\left(\prod_{\alpha>0} \frac{[(\alpha, \Lambda+\rho)]_{q}}{[(\alpha, \rho)]_{q}}\right) .
$$

Proof. The middle product factor here is Weyl's formula for $\operatorname{dim} V(\Lambda)$. Continuing from Proposition 2.4 we use the fact that the bilinear form on $t^{*}$ is symmetric and invariant under the Weyl group to write $J(\Lambda+\rho)(2 \rho)=\sum_{w}(\operatorname{det} w) q^{(w(\Lambda+\rho), 2 \rho)}=$ $\sum_{w}(\operatorname{det} w) q^{\left(w^{-1} \rho, 2(\Lambda+\rho)\right)}=\sum_{w}(\operatorname{det} w) q^{(w \rho, 2(\Lambda+\rho))}=J(\rho)(2(\Lambda+\rho))$ which is just $q^{(\rho, 2(\Lambda+\rho))}$. 
$\prod_{\alpha>0}\left(1-q^{-2(\alpha, \Lambda+\rho)}\right)$. Recalling that $\rho$ is the half-sum of the positive roots, we have $\prod_{\alpha>0}^{\alpha>0}\left(q^{(\alpha, \Lambda+\rho)}-q^{-(\alpha, \Lambda+\rho)}\right)$ as required. Similarly for the denominator $J(\rho)(2 \rho)$.

We remark that the last factor in Proposition 2.5 was found useful in [1] and called the " $q$-dimension" of $V(\Lambda)$ in analogy with Weyl's formula. The intrinsic category-theoretic $\operatorname{rank}(V(\Lambda))$ of Proposition 1.1 is different, having the further gaussian factor which will be crucial in what follows.

\section{Computation of Rank}

In this section we prove the main theorem, Theorem 3.2. This asserts that $\operatorname{rank}\left(U_{q}(g)\right)$ is a theta function associated to the weight lattice. The result precisely generalizes the result for $\operatorname{rank}\left(U_{q}(\operatorname{su}(2))\right)$.

Lemma 3.1. Let $d(\Lambda)=\prod_{\alpha>0} \frac{(\alpha, \Lambda)}{(\alpha, \rho)}$ for $\Lambda \in t^{*}$. Let

$$
d^{(\mu)}(\Lambda)=\left(\frac{\partial^{|\mu|} d}{\partial^{\mu_{1}} \omega_{1} \partial^{\mu_{2}} \omega_{2} \cdots \partial^{\mu_{l}} \omega_{l}}\right)(\Lambda) .
$$

Here $\mu \in P=\left\{\mu \in t^{*} \mid\left(\mu, \check{\alpha}_{i}\right) \in \mathbb{Z}\right\}$ is the weight lattice with basis of fundamental weights $\left\{\omega_{i}\right\}$ over $\mathbb{Z}$, and $\mu_{i}$ are the components of $\mu$ in this basis. $|\mu|=\sum_{i} \mu_{i}$. Let $N=\operatorname{card}\{\alpha \mid \alpha>0\}$ and $\mu !=\prod_{i}\left(\mu_{i} !\right)$. Then

$$
\operatorname{rank}\left(U_{q}(g)\right)=\frac{\sum_{|\mu| \leqq N}(\mu !)^{-1} \sum_{\Lambda \in P} d^{(\mu)}(\Lambda) q^{-\|\Lambda\|^{2}}}{\prod_{\alpha>0}\left(1-q^{-2(\alpha, \rho)}\right)} .
$$

Proof. Since $W$ acts by isometries we have $d(w \Lambda)=\operatorname{det}(w) d(\Lambda)$ for $w \in W$. Also, if $\Lambda, \mu \in P_{+}, w_{1}, w_{2} \in W$ and $w_{1}(\Lambda+\rho)=w_{2}(\mu+\rho)$ then $\Lambda=\mu$. From Proposition 2.4 and the proof of Proposition 2.5, we have

$$
\operatorname{rank}\left(U_{q}(g)\right)=\frac{\sum_{w \in W} \sum_{\Lambda \in P_{+}} d(w(\Lambda+\rho)) q^{-\|w(\Lambda+\rho)-\rho\|^{2}}}{\prod_{\alpha>0}\left(1-q^{-2(\alpha, \rho)}\right)} .
$$

$\frac{\partial}{\partial \omega_{i}}$ in $d^{(\mu)}$ denotes differentiation in direction $\omega_{i}$ in $t^{*}$. The numerator here is therefore the same as

$$
\sum_{\Lambda \in P} d(\Lambda) q^{-\|\Lambda-\rho\|^{2}}=\sum_{\Lambda \in P} d(\Lambda+\rho) q^{-\|\Lambda\|^{2}}=\sum_{|\mu| \leqq N}(\mu !)^{-1} \sum_{\Lambda \in P} d^{(\mu)}(\Lambda) q^{-\|\Lambda\|^{2}} .
$$
Theorem 3.2. Let $f_{\mu}=(\mu !)^{-1} \sum_{\Lambda \in P} d^{(\mu)}(\Lambda) q^{-\|\Lambda\|^{2}}$ as in the previous lemma. Then $f_{\mu}=0$
for all $|\mu|<N$ and

$$
\operatorname{rank}\left(U_{q}(g)\right)=\frac{\sum_{\Lambda \in P} q^{-\|\Lambda\|^{2}}}{\prod_{\alpha>0}\left(1-q^{-2(\alpha, \rho)}\right)} .
$$


Proof. We use notations and results of [10, Chap. 3]. Let $H_{k}(W)$ denote the space of $W$-harmonic homogeneous polynomials of degree $k$ on $\mathbb{C} \otimes P$. W-harmonic here means in particular harmonic with respect to $\Delta^{*}$, where $\Delta$ is the Laplace operator corresponding to (,). Let $I_{+}(W)$ be the ideal of $W$-invariant polynomials $\phi$ on $\mathbb{C} \otimes P$ such that $\phi(0)=0$, and $\partial\left(I_{+}\right)$the corresponding ring of differential operators. $f \in H_{k}(W)$ means that $\partial\left(I_{+}\right) f=0$. It is known that $H_{k}(W) \cap I_{+}(W)=0$ if $k>0$, see [10, Theorem 3.4]. It is also known that $H_{k}(W)=\operatorname{span}\left\{d^{(\mu)}\right\}_{|\mu|=N-k}$, see [10, Theorem 3.6(i)]. Hence $\sum_{w \in W} d^{(\mu)}(w \Lambda)=0$ if $|\mu|<N$. Now, card $(W) \sum_{\Lambda \in P} d^{(\mu)}(\Lambda)$. $q^{-\|\Lambda\|^{2}}=\operatorname{card}(W) \sum_{\Lambda \in P} d^{(\mu)}(w \Lambda) q^{-\|w \Lambda\|^{2}}=\sum_{w \in W} \sum_{\Lambda \in P} d^{(\mu)}(w \Lambda) q^{-\|\Lambda\|^{2}}$. Hence $f_{\mu}=0$ for $|\mu|<N$. Finally, it is easy to see that $\sum_{|\mu|=N}(\mu !)^{-1} d^{(\mu)}(\Lambda)=d(\rho)=1$. The result then
follows from Lemma 3.1.

\section{Modular Properties}

For computations it is useful to evaluate the above formula explicitly in terms of the Cartan matrix for $g$. Proposition 2.5 is then,

Proposition 4.1. Let $g$ be a complex simple Lie algebra with rankl, a the Cartan matrix and $\alpha_{i}$ simple roots as above. Let $b$ be the vector given from the lengths by $b_{i}=\frac{1}{2}\left\|\alpha_{i}\right\|^{2}$ and let $A_{i j}=b_{i} a_{i j}^{-1}, p_{j}=\sum_{i} b_{i} a_{i j}^{-1}$. Then

$$
\operatorname{rank}\left(U_{q}(g)\right)=\sum_{n \in \mathbb{Z}_{+}^{l}} q^{-n^{T} A n-2 p \cdot n} \prod_{m \in R_{+}} \frac{d(m \cdot b+m \cdot(n b))}{d(m \cdot b)}
$$

where $d(x)=\left(q^{x}-q^{-x}\right) x$. Here $m$ are the $\mathbb{Z}_{+}$-valued vectors corresponding to positive roots $\sum_{i} m_{i} \alpha_{i}$. We have used the usual vector notations for the Euclidean inner product and for transpose. $n b$ is given by the product $(n b)_{i}=n_{i} b_{i}$.

Proof. $P_{+}$is the half-lattice generated over $\mathbb{Z}_{+}$by basis $\omega_{i} \in t^{*}$ such that $\left(\omega_{i}, \check{\alpha}_{j}\right)=\delta_{i j}$. Hence $\omega_{i}=\sum_{j} a_{j i}^{-1} \alpha_{j}$, giving $(\Lambda, \Lambda)$. For $(\Lambda, 2 \rho)$ use $\left(\omega_{i}, \rho\right)=\sum_{j} a_{j i}^{-1} \frac{1}{2}\left\|\alpha_{j}\right\|^{2}\left(\check{\alpha}_{j}, \rho\right)$ and $\left(\check{\alpha}_{j}, \rho\right)=1$ for all $j$ since $\rho$ is the half-sum of positive roots. Similar computations give the $(\alpha, \rho)$ and $(\alpha+\Lambda, \rho)$.

We see from this expression that $\operatorname{rank}\left(U_{q}(g)\right)$ is a sum of derivatives of rank $g$-dimensional theta functions (completing squares and differentiating with respect to a linear term in the exponent to bring down $n_{i}$ factors). The content of Theorem 3.2 is that these terms exhibit remarkable cancellations among theselves to give a rank $g$-dimensional theta function. Theorem 3.2 is,

Proposition 4.2. Let $g$ be a complex simple Lie algebra of rankl and $A$ the matrix defined from the vector of square lengths $b$ and the inverse Cartan matrix as in Proposition 4.1. $R_{+}$denotes the positive root vectors. Then

$$
\operatorname{rank}\left(U_{q}(g)\right)=\frac{\sum_{n \in \mathbb{Z}^{l}} q^{-n^{T} A n}}{\prod_{m \in R_{+}}\left(1-q^{-2 m \cdot b}\right)} .
$$


Example 4.3 [19]. For $g=s u(2)$ we take $a=(2)$ and $b=(1)$. There is only one positive root, $\alpha_{1}$, and $\rho=\frac{1}{2} \alpha_{1}$. The remarkable cancellations in $\operatorname{rank}\left(U_{q}(\operatorname{su}(2))\right)$ can be seen directly: $\operatorname{rank}\left(U_{q}(\operatorname{su}(2))\right)=\sum_{n \in \mathbb{Z}_{+}} q^{-n((n / 2)+1)}\left(q^{n+1}-q^{-n-1}\right)(n+1) /\left(q-q^{-1}\right)=$ $\sum_{n \in \mathbb{Z}_{+}}\left((n+1) q^{-n^{2} / 2}-((n+3)-2) q^{-(n+2)^{2} / 2}\right) /\left(1-q^{-2}\right)=\left(1+2 \sum_{n \in \mathbb{N}} q^{-n^{2} / 2}\right) /\left(1-q^{-2}\right)$. Because of this cancellation of terms the coefficients of $q^{-n^{2 / 2}}$ in the numerator are 2 rather than growing as $n$.

A corollary of Theorem 3.2 and Proposition 4.2 is a result motivated by the conjecture of Sect. 2,

Corollary 4.4. Let $g$ be a complex simple Lie algebra. Then $\operatorname{rank}\left(U_{q}(g)\right)$ has an expression as sums and products of one-dimensional theta functions $\theta_{\delta}(q)=\sum_{n \in \mathbb{Z}} q^{(n+\delta)^{2}}$, where $\delta \in \mathbb{Q}$. In this form, $\operatorname{rank}\left(U_{q}(g)\right)$ vanishes for all $q$ a rational root of unity.

Proof. $A$ has rational coefficients. We can complete the square in $n^{T} A n$ for $n_{1}$, writing $n^{T} A n=\beta_{1}\left(n_{1}+f_{1}\left(n_{2}, \ldots, n_{l}\right)\right)^{2}+g\left(n_{2}, \ldots, n_{l}\right)$. Here $f_{1}$ is linear and $g$ quadratic in the $n_{2}$ to $n_{l}$, both with rational coefficients, and $\beta_{1}$ is rational. Iterating this we have $n^{T} A n=\beta_{1}\left(n_{1}+f_{1}\left(n_{2}, \ldots, n_{l}\right)\right)^{2}+\beta_{2}\left(n_{2}+f_{2}\left(n_{3}, \ldots, n_{l}\right)\right)^{2}+\cdots+\beta_{l} n_{l}^{2}$. Now split the values of $n_{2} \cdots n_{l}$ into various modulo classes, in such a way that in each class the value of the fractional part of $f_{1}$ is a constant, say $\delta_{1}$. In each class, the $n_{1}$ sum then gives a $\theta_{\delta_{1}}\left(q^{-\beta_{1}}\right)$. However, the sum over $n_{2}, \ldots, n_{l}$ within each class is itself expressible as a sum over all integers $n_{2}^{\prime}, \ldots, n_{l}^{\prime}$, say. Hence in each class we can repeat the above for $n_{2}^{\prime}$. The result then follows by iteration.

Example 4.5. For $g=s u(3)$ we have $a=\left(\begin{array}{cc}2 & -1 \\ -1 & 2\end{array}\right)$ and $b=(1,1)$. We have in Proposition 4.2, $\operatorname{rank}\left(U_{q}(s u(3))\right)=\sum_{m, n \in \mathbb{Z}} q^{-(2 / 3)\left(m^{2}+n^{2}+m n\right)} /\left(1-q^{-2}\right)^{2}\left(1-q^{-4}\right)$. Following the method of Corollary 4.4 the numerator is $\sum_{m, n \in \mathbb{Z}} q^{-(2 / 3)\left((m+(1 / 2) n)^{2}+(3 / 4) n^{2}\right)}=$ $\sum_{k \in \mathbb{Z}} q^{-2 k^{2}} \sum_{m \in \mathbb{Z}} q^{-(2 / 3) m^{2}}+\sum_{k \in \mathbb{Z}} q^{-2(k+(1 / 2))^{2}} \sum_{m \in \mathbb{Z}} q^{-(2 / 3)(m+(1 / 2))^{2}}$. With $\theta=\theta_{0}$ we have,

$$
\operatorname{rank}\left(U_{q}(\operatorname{su}(3))\right)=\frac{\theta\left(q^{-2}\right) \theta\left(q^{-2 / 3}\right)+\theta_{1 / 2}\left(q^{-2}\right) \theta_{1 / 2}\left(q^{-2 / 3}\right)}{\left(1-q^{-2}\right)^{2}\left(1-q^{-4}\right)} .
$$

Similarly,

$$
\operatorname{rank}\left(U_{q}(\operatorname{su}(4))\right)=\frac{\left(\theta\left(q^{-2}\right)^{2}+\theta_{1 / 2}\left(q^{-2}\right)^{2}\right) \theta\left(q^{-1}\right)+2 \theta\left(q^{-2}\right) \theta_{1 / 2}\left(q^{-2}\right) \theta_{1 / 2}\left(q^{-1}\right)}{\left(1-q^{-2}\right)^{3}\left(1-q^{-4}\right)^{2}\left(1-q^{-6}\right)} .
$$

Corollary 4.6. Conjecture 2.1 holds for $g=s u(3), s u(4)$.

Proof. Note that $\theta_{\delta}(q)=q^{\delta^{2}} \prod_{n=1}^{\infty}\left(1-q^{2 n}\right)\left(1+q^{2(n+\delta)-1}\right)\left(1+q^{2(n-\delta)-1}\right)$ (a form of Jacobi's triple product identity). Hence every term in the numerators in Example 4.5 contains the factor $\prod_{n=1}^{\infty}\left(1-q^{-n}\right)$. Similar results apply for all the $\operatorname{rank}\left(U_{q}(\operatorname{su}(l+1))\right)$.

As another corollary of Theorem 3.2 and Proposition 4.2 it follows that the numerators in $\operatorname{rank}\left(U_{q}(g)\right)$ are modular forms with respect to a suitable subgroup of $S L(2, \mathbb{Z})$ [14, Corollary 13.6]. We have for example, 
Proposition 4.7. Let $e_{8}$ denote the exceptional Lie algebra of the corresponding Dynkin diagram. Then the numerator of $\operatorname{rank}\left(U_{q}\left(e_{8}\right)\right)$ is a modular form of weight 4. Explicitly, rank $\left(U_{q}\left(e_{8}\right)\right)$ has as numerator the modular form $E_{2}\left(q^{-2}\right)=$ $1+240 \sum_{n \in \mathbb{Z}} r_{n} q^{-2 n}$, where $r_{n}=\sum_{d / n} d^{3}$. The sum in $r_{n}$ is over positive divisors $d$.

Proof. $e_{8}$ has all roots of square length 2, so $A$ in Proposition 4.2 is the inverse of the Cartan matrix $a$ for $e_{8}$. As is well known [21, V.1.4.3], this Cartan matrix has determinant 1 and hence defines a unimodular lattice $\Gamma$. Hence $\theta_{\Gamma}(z) \equiv \sum_{n_{i} \in \mathbb{Z}} e^{\pi i z n^{T} a n}$ is a modular form, which turns out to be $E_{2}(z)$ [21, VII.6.6]. Hence $\theta_{\Gamma}(z)=$ $(l z)^{4} \theta_{\Gamma}\left(-\frac{1}{z}\right)=\theta_{\Gamma^{\prime}}(z)$, the last equality being the Jacobi inversion formula. Hence $E_{2}(z)=\sum_{n_{i} \in \mathbb{Z}} e^{\pi l z n^{T} A n}$ also. Regarding this as a function of $q=e^{2 \pi l z}$ and using its known $q$ expansion [21, VII.4.1], gives the result stated.

The remaining result of this section is motivated by the properties of the coefficients of the Dedekind $\eta$ function and other modular forms. Write

$$
\eta(q)^{24}=q \prod_{n=1}^{\infty}(1-q)^{24}=\sum_{n=1}^{\infty} \tau_{n} q^{n}
$$

where the coefficients $\tau_{n}$ are Ramanujan's $\tau$ function on $\mathbb{N}$. This has the property $[21$, VII.4.5],

$$
\tau_{m} \tau_{n}=\sum_{d \mid(m, n)} d^{11} \tau_{m n / d^{2}}
$$

Here the sum is over common divisors $d$ of $m, n$. In particular, $\tau$ is said to be multiplicative in the following sense: if $m$ and $n$ are coprime then $\tau_{m} \tau_{n}=\tau_{m n}$. If a modular form $f(q)=f(0)+\sum_{n \in \mathbb{N}} f_{n} q^{n}$ is of positive weight and a simultaneous eigenfunction of the Hecke operators $T_{n}$ then its coefficients $f_{n}$ are multiplicative [21, VII.5.4]. (Here we assume normalization such that $f_{1}=1$.) $E_{2}$ in Proposition 4.7 is an example. Thus this property of the coefficients when $m, n$ are coprime is associated with modular functions in some generality and provides an alternative to summing up the expression for $\operatorname{rank}\left(U_{q}(g)\right)$.

This multiplicativity property clearly holds for coefficients of the numerator of the rank of quantum $s u(2)$ in the form

$$
\operatorname{rank}\left(U_{q^{-2}}(\operatorname{su}(2))\right)=\frac{1+2 r(q)}{1-q^{4}}, \quad r(q)=\sum_{n \in \mathbb{N}} q^{n^{2}} .
$$

If $n, m$ are coprime then the coefficients obey $r_{m n}=r_{m} r_{n}$. This is because these coefficients are non-zero only on perfect squares, where they have value 1 : if $m$ and $n$ have no non-trivial common factor and are perfect squares, $m n$ is also a perfect square, while if $n$ or $m$ is not a perfect square then since there are no non-trivial common factors, neither is $m n$.

Proposition 4.8. Let $r$ be the numerator function in

$$
\operatorname{rank}\left(U_{q^{-3 / 2}}(s u(3))\right)=\frac{1+6 r(q)}{\left(1-q^{3}\right)^{2}\left(1-q^{6}\right)} .
$$


If $m, n$ are coprime then $r_{m n}=r_{m} r_{n}$, i.e. the coefficients are multiplicative. The value on prime powers is

$$
r_{p^{n}}= \begin{cases}1 & p=3 \\ n+1 & p \equiv 1(3) \\ 0 & n \text { odd }, p \equiv 2(3) \\ 1 & n \text { even, } p \equiv 2(3)\end{cases}
$$

for all primes $p$ and $n \in \mathbb{N}$. Here (3) means modulo 3 .

Proof. The function $1+6 r(q) \equiv 1+6 \sum_{n \in \mathbb{N}} r_{n} q^{n}=\sum_{n_{1}, n_{2} \in \mathbb{Z}} q^{n_{1}^{2}+n_{2}^{2}+n_{1} n_{2}}$. So $6 r_{n}$ is the number of integers $\left(n_{1}, n_{2}\right)$ such that the quadratic form $A\left(n_{1}, n_{2}\right)=n_{1}^{2}+n_{2}^{2}+n_{1} n_{2}$ has value $n$. This is a well studied subject in number theory and it is known that $6 r_{n}=6 \sum_{m / n}\left(\frac{-3}{m}\right)$. Here $\left(\frac{-3}{m}\right)$ is the Legendre symbol extended to include $m=2$ and non-primes. See e.g. [3, Chap. 6] (where the formula is given for general binary quadratic forms). In the present case

$$
\begin{aligned}
\left(\frac{-3}{p}\right)= & \begin{cases}0 & p=0 \\
1 & \text { if } x^{2}+3 \equiv 0(p) \text { has a solution } \\
-1 & \text { if not }\end{cases} \\
& = \begin{cases}0 & p=3 \\
1 & p \equiv 1(3) \\
-1 & p \equiv 2(3)\end{cases}
\end{aligned}
$$

for all primes $p$, extended multiplicatively. The first equality is the definition for $p>2$. The second is Gauss' law of quadratic reciprocity, $\left(\frac{-3}{p}\right)=\left(\frac{p}{3}\right)$ and provides the definition when $p=2$. This then immediately gives the result for $r$ stated. Multiplicativity of $r_{n}$ follows from multiplicativity of $\left(\frac{-3}{m}\right)$. The result was first
obtained by computer.

From this proposition it is easy to recognize the numerator in $\operatorname{rank}\left(U_{q-(3 / 2)}(\operatorname{su}(3))\right)$ as follows. For every multiplicative function $f(q)=\sum_{n \in \mathbb{N}} f_{n} q^{n}$, the Mellin transform takes the form $\tilde{f}(s)=\sum_{n \in \mathbb{N}} f_{n} n^{-s}=\prod_{p}\left(1+\sum_{n=1}^{\infty} f_{p^{n}} p^{-n s}\right)$, where the product is over primes. For example, the Mellin transform of a theta function is $\zeta(2 s)$, where $\zeta$ is a Riemann $\zeta$ function, $\zeta(s)=\prod_{p}\left(1-p^{-s}\right)^{-1}$. In the present case we have
immediately

$$
\tilde{r}(s)=\left(1-3^{-s}\right)^{-1} \prod_{p \equiv 1(3)}\left(1-p^{-s}\right)^{-2} \prod_{p^{\prime} \equiv 2(3)}\left(1-p^{\prime-2 s}\right)^{-1}=\zeta(s) L(s, \chi),
$$

where $L(s, \chi)=\sum_{n=1}^{\infty} \chi(n) n^{-s}=\prod_{p}\left(1-\chi(p) p^{-s}\right)^{-1}$ is the $L$-function associated to the non-trivial character of the Galois group for the field extension $\mathbb{Q}\left[e^{2 \pi / 3}\right]$ of $\mathbb{Q}$ by a cube root of unity. The Galois group is defined as automorphisms of the extended field that fix the base field. In the present case it has two elements, $\sigma_{1}$ the identity 
and $\sigma_{2}: e^{2 \pi \imath / 3} \mapsto\left(e^{2 \pi / 3}\right)^{2}$. The non-trivial character sends $\sigma_{1} \mapsto 1, \sigma_{2} \mapsto-1$. This can be viewed as a function $\chi$ on natural numbers that are prime to 3 (extended to have value zero otherwise), giving in the present case $\chi(p)=\left(\frac{p}{3}\right)[21$, VI.1.3]. This yields the same Euler product for $\zeta(s) L(s, \chi)$ as that obtained for $\tilde{r}$. The Euler product (4) for $\tilde{r}$ was also obtained independently by very different means in [11, Appendix]. In our case it follows easily from multiplicativity in Proposition 4.8.

Note that the trivial character $\chi=1$ also defines an $L$-function, $L(s, 1)=$ $\left(1-3^{-s}\right) \zeta(s)$, so the numerator function $r$ in $\operatorname{rank}\left(U_{q^{-3 / 2}}(s u(3))\right)$ has Mellin transform $\left(1-3^{-s}\right)^{-1} \prod L(s, \chi)$. For comparison, rank $\left(U_{q^{-2}}(s u(2))\right)$ has numerator function $r=\sum_{n \in \mathbb{N}} q^{n^{2}}$ with Mellin transform $\zeta(2 s)$ while the associated field extension is trivial, so that the only character is trivial, with $L(s, 1)=\left(1-2^{-s}\right) \zeta(s)$. The numerator functions for higher $\operatorname{rank}\left(U_{q}(s u(l+1))\right)$ have also been investigated. They have interesting but more complicated number theoretic properties.

\section{Concluding Remarks}

We conclude with some remarks about directions for further work. Firstly, a possible physical interpretation of the main theorem, Theorem 3.2, is as follows. It will be discussed in more detail elsewhere. The left-hand side of the theorem is the rank, which is $\operatorname{Tr} \underline{u}$ in the left regular representation. This $\underline{u}$ is well known in the construction of link invariants from quantum groups, e.g. [20]. Previously, only finite-dimensional representations have been considered. It is also well-known since [23] that these invariants can apparently be interpreted as the expectation value of Wilson loops in a quantum field theory with Chern-Simons Lagrangian $\frac{k}{4 \pi} \int_{S^{3}} \operatorname{Tr} A d A+\frac{2}{3} A^{3}$ ( $k$ an integer). The quantum theory here has to be suitably interpreted, for example by using point-splitting or by working with ribbons. In particular we have for the vacuum expectation value of a Wilson loop of a trivial knot $\kappa$ with framing one,

$$
\frac{\left\langle 0\left|\operatorname{Tr} P_{\rho} e_{\kappa}^{\int A}\right| 0\right\rangle}{\langle 0 \mid 0\rangle}=\operatorname{Tr} \rho(\underline{u}) .
$$

Here $P_{\rho}$ denotes path-ordered integration in representation $\rho$ of the Lie algebra $g$. On the right $\rho$ is the canonical deformation of this to a representation of $U_{q}(g)$ and $q$ has to be specialized to $q=e^{(2 \pi i) /\left(k+g_{c}\right)}$, where $g_{c}$ is the dual Coxeter number of $g$. Thus in particular, $\operatorname{rank}\left(U_{q}(g)\right)$ is basically the vacuum expectation value of the unknot in $\rho$ the left regular representation in the Chern-Simons theory. This is an interpretation of the left-hand side of Theorem 3.2.

On the other hand we can interpret the numerator on the right-hand side of Theorem 3.2 for $\operatorname{rank}\left(U_{q}(g)\right)$ basically as the partition function for a quantum particle confined to a certain bounded domain in Euclidean space, namely an alcove of $g$ [2]. For example, the numerator for 
$\operatorname{rank}\left(U_{q}(\operatorname{su}(3))\right)$ is

$$
\sum_{m, n \in \mathbf{Z}} q^{-(2 / 3)\left(m^{2}+n^{2}+m n\right)}=1+6 \sum_{m \geqq 0, n>0} q^{-(2 / 3)\left(m^{2}+n^{2}+m n\right)}
$$

while the energy spectrum for a quantum particle of mass $M$ confined to an equilateral triangle of side $a$ (with wave function vanishing on the boundary) is $\frac{\hbar^{2}}{2 M}\left(\frac{4 \pi}{3 a}\right)^{2}\left(n^{2}+m^{2}+n m\right)$ for $n, m>0$. This is a classic problem, but see for example [11]. Thus the numerator function of $\operatorname{rank}\left(U_{q}(s u(3))\right)$ is basically the partition function for this system with

$$
q=e^{\left(4 \pi^{2} / 3 a^{2}\right)\left(\hbar^{2} / M k T\right)},
$$

where $T$ is the temperature and $k$ is now Boltzmann's constant. Thus just as $|G|$ counts the points in a classical set $G$, the quantum group rank counts the "points" in $U_{q}(g)$ in a quantum mechanical sense.

Accordingly, Theorem 3.2 relates these two very different physical systems. Note that the rank invariant studied in this paper is only the simplest in a family of quasitriangular Hopf algebra invariants indexed by knots and links $\kappa$. Let $\Lambda \in P_{+}$ be a dominant weight and $I_{\Lambda}\left(\kappa, U_{q}(g)\right)$ be the link invariant obtained by standard quantum group methods (or the Chern-Simons theory) for the representation $V(\Lambda)$. Then we are led to consider

$$
I\left(\kappa, U_{q}(g)\right)=\sum_{\Lambda \in P_{+}}(\operatorname{dim} V(\Lambda)) I_{\Lambda}\left(\kappa, U_{q}(g)\right)
$$

for any knot or link $\kappa$. The rank is the invariant corresponding to the unknot. It seems reasonable to expect that the higher members of the family would like-wise have interesting number-theoretic properties as functions of $q$ and an interpretation in terms of quantum mechanics in an alcove of $g$.

Finally, we note that the rank theory for quasitriangular Hopf algebras extends easily to quasitriangular quasi-Hopf algebras along the lines of Sect. 1. Let $g$ be a simple Lie algebra and let $A$ be a quasitriangular quasi-Hopf algebra [7] over $\mathbb{C}[[\hbar]]$ quantizing the universal enveloping algebra $U(g)$, i.e. such that $A$ is a topologically free $\mathbb{C}[[\hbar]]$-module and $A / \hbar A \cong U(g)$. Then $\operatorname{rank}(A)=\operatorname{rank}\left(U_{q}(g)\right)$ (where $q=e^{\hbar / 2}$ ). The proof of this depends on the fact that the rank of a quasitriangular quasi-Hopf algebra is invariant under the "twisting" or "gauge transformation" introduced in [7]. It is already known (as a case of [7, Theorem 1]) that every quantization is obtained by a twisting of $U_{q}(g)$.

In this context we also observe that there is another independent connection between braid invariants and Galois theory, and this was recently connected with quasitriangular quasi-Hopf algebras in [8]. It may be very interesting to try to relate this with our results above. The preprint arrived some time after the present manuscript was completed: we mention it as an interesting possibility for further work.

Acknowledgements. S. M. would like to thank M. J. Rodriguez for discussions at an early stage. We would also like to thank R. Pinch for the advice that modular functions could be thought of in terms of multiplicativity of the coefficients. The observations centered on Eq. (4) grew out of our joint discussion. Finally, thanks to A. J. Macfarlane for valuable discussions on the interpretation. 


\section{References}

1. Alvarez-Gaumé, L., Gomez, C., Sierra, G.: Duality and quantum groups. Nucl. Phys. B330, 347 (1990)

2. Bérard, P.: Spectres et groupes cristallographiques. C. R. Acad. Sci., Paris, Ser. A, 288, 1059-1060 (1979)

3. Davenport, H.: Multiplicative Number Theory. Berlin, Heidelberg, New York: Springer 1980

4. Deligne, P., Milne, J. S.: Tannakian categories. Lecture Notes in Mathematics, vol. 900. Berlin, Heidelberg, New York: Springer 1982

5. Drinfeld, V. G.: Quantum groups. In: Gleason, A. (ed.). Proceedings of the ICM, pp. 798-820. Providence, Rhode Island: AMS 1987

6. Drinfeld, V. G.: On quasi-cocommutative Hopf algebras. Algebra i Analiz 2, 30-46 (1989) In Russian

7. Drinfeld, V. G.: Quasi-Hopf algebras and Knizhnik-Zamolodchikov equations. Acad. Sci. Ukr. preprint, ITP-89-43E, 1989

8. Drinfeld, V. G.: On quasitriangular quasi-Hopf algebras and a certain group closely connected with $\mathrm{Gal}(\overline{\mathbb{Q}} / \mathbb{Q})$. Preprint, 1990. In Russian

9. Freyd, P., Yetter, D.: Braided compact closed categories with applications to low dimensional topology. Adv. Math. 77, 156-182 (1989)

10. Helgason, S.: Groups and geometric analysis, integral geometry, invariant differential equations and spherical functions. New York: Academic 1984

11. Itzykson, C., Luck, J. M.: Arithmetical degeneracies in simple quantum systems. J. Phys. A 9, 211-239 (1986)

12. Jimbo, M.: A $q$-difference analog of $U(g)$ and the Yang-Baxter equation. Lett. Math. Phys. 10, 63-69 (1985)

13. Joyal, A., Street, R.: Braided monoidal categories. Mathematics Reports 86008, Macquaire University, 1986

14. Kac, V. G.: Infinite dimensional Lie algebras. CUP: Cambridge 1985

15. Larson, R. G., Radford, D. E.: Semisimple cosemisimple Hopf algebras. Am. J. Math. 110, $187-195(1988)$

16. Lusztig, G.: Modular representations and quantum groups. Contemp. Math. 82, 59-78 (1989)

17. MacDonald, I. G.: Affine root systems and dedekind's $\eta$-function. Invent. Math. 15, 91-143 (1972)

18. Majid, S.: Representation-theoretic rank and double Hopf algebras. Comm. Algebra. 18, 3705-3712 (1990)

19. Majid, S.: Quasitriangular Hopf algebras and Yang-Baxter equations. Int. J. Mod. Phys. A 5(1), 1-91 (1990)

20. Reshetikhin, N. Yu., Turaev, V. G.: Ribbon graphs and their invariants derived from quantum groups. Commun. Math. Phys. 127(1), 1-26 (1990)

21. Serre, J. P.: A course in arithmetic. Berlin, Heidelberg, New York: Springer 1970

22. Soibelman, Ya. S.: Quantum Weyl group and some of its applications. Suppl. Rend. Circ. Mat. Palermo, 1990. (To appear)

23. Witten, E.: Some geometric applications of quantum field theory. In: Proc. ICMP, Swansea, 1988. Adam Hilger

Communicated by N. Yu. Reshetikhin 